\title{
Dual Current-Mode Control for Single-Switch Two-Output Switching Power Converters
}

\author{
S. C. Wong, C. K. Tse and K. C. Tang \\ Department of Electronic and Information Engineering \\ Hong Kong Polytechnic University, Hunghom, Hong Kong, China
}

\begin{abstract}
A novel method for programming current in dc/dc converters operating in discontinuous conduction mode is described in this paper. The control variable is the product of the square of the duty cyle and the switching period, i.e., $D^{2} T$, which is directly proportional to input and output currents of a discontinuous-mode converter. A method of controlling $D^{2} T$ is applied to converters that utilize one switch (or one set of synchronous switches) for achieving two control functions. In particular a single-switch two-output boost converter, in which a continuous-mode converter and a discontinuous-mode converter share one active switch, is studied. In this system, current-mode control is used to regulate the output voltage of the continuousmode converter and the proposed $D^{2} T$ control is used to regulate the other discontinuous-mode converter. The result is a generic current-mode controlled dual-output converter.

Keywords-Current-mode control, dc/dc converter, dual-output converter, discontinuous conduction mode.
\end{abstract}

\section{INTRODUCTION}

This paper describes a single-switch two-output regulator which comprises two boost converters, one operating in continuous conduction mode (CCM) and the other in discontinuous conduction mode (DCM) [1], [2]. Previously reported control methods take advantage of the insensitivity of the CCM converter to switching frequency, and apply duty-cycle control to regulate the CCM converter while regulating the DCM converter by frequency modulation [3], [4]. In this paper, we consider application of generic current-mode control to both converters in order to achieve faster transient responses [5]. Specifically, we use a standard current programming for the CCM converter and a novel $D^{2} T$ control for regulating the DCM converter. We will also discuss the local stability of the combined current-mode and $D^{2} T$ control scheme, and present a practical circuit implementation of the controller.

\section{Outline of the Proposed Control}

Inspired by its averaged behaviour model, the DCM converter can be controlled by varying the control quantity $D^{2} T$ which is proportional to the output current [6]. In principle, if the quantity $D^{2} T$ is adjusted via a feedback mechanism, the output load voltage can be regulated. A special but practically important case is when either the on-time or off-time duration is already determined by another control law. We shall make reference to a single-switch two-output boost converter later in the paper.

Suppose the duty cycle is $D$, and the period begins with the switch turned off. Thus, after a duration of $(1-D) T$, the switch is turned on; and after another duration of $D T$, the switch is turned off again, completing one cycle.

Our objective is to derive a scheme, whereby the off-time duration $(1-D) T$ is pre-determined and the period $T$ is adjusted to give the desired value of $D^{2} T$. The proposed

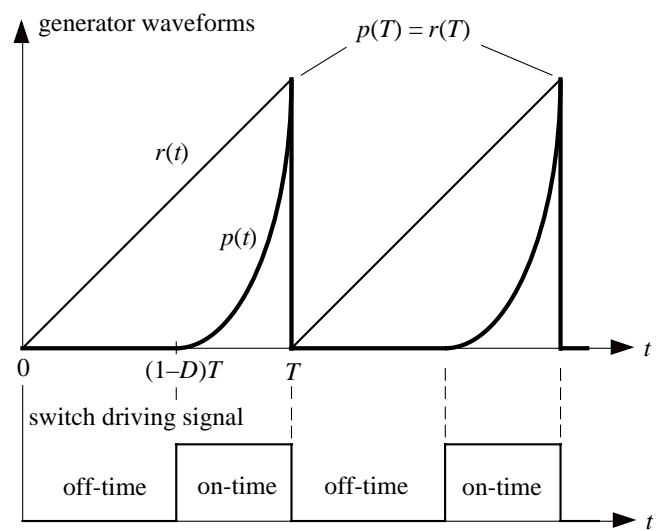

(a)

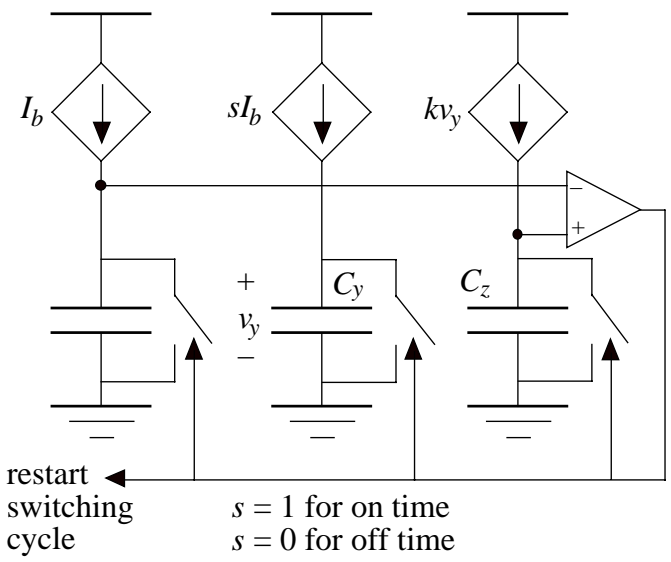

(b)

Fig. 1. (a) Waveforms of the parabolic and ramp generators in the conceptual $D^{2} T$ control scheme; (b) conceptual implementation.

controller consists of a periodic parabola generator $p(t)$ and a ramp generator $r(t)$ :

$$
\begin{aligned}
& p(t)=a\left(t-t_{0}\right)^{2} \\
& r(t)=b\left(t-t_{0}^{\prime}\right)
\end{aligned}
$$

where $t_{0}$ and $t_{0}^{\prime}$ are arbitrary start instants for the two generators, and $a$ and $b$ can be considered as constant for the time being. Fig. 1 (a) shows these generator waveforms.

At the start of the switching cycle, say $t=0$, the ramp generator is triggered to start. At $t=(1-D) T$ (which is determined externally), the switch is turned on. At the same time, the parabola generator is triggered to start from zero. As soon as the outputs of the generators are equal, the switch is turned off whereby spawning a new cycle. Clearly, this 


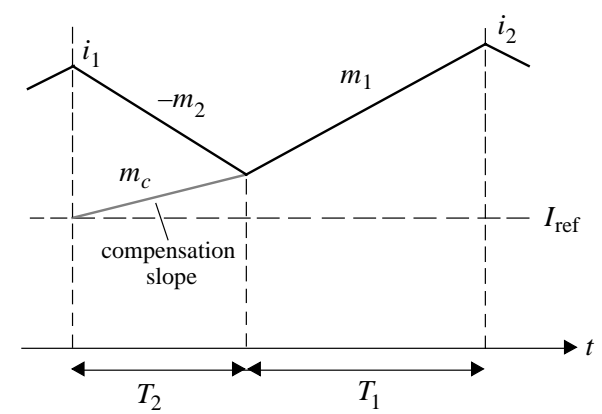

Fig. 2. Inductor current waveform for stability analysis.

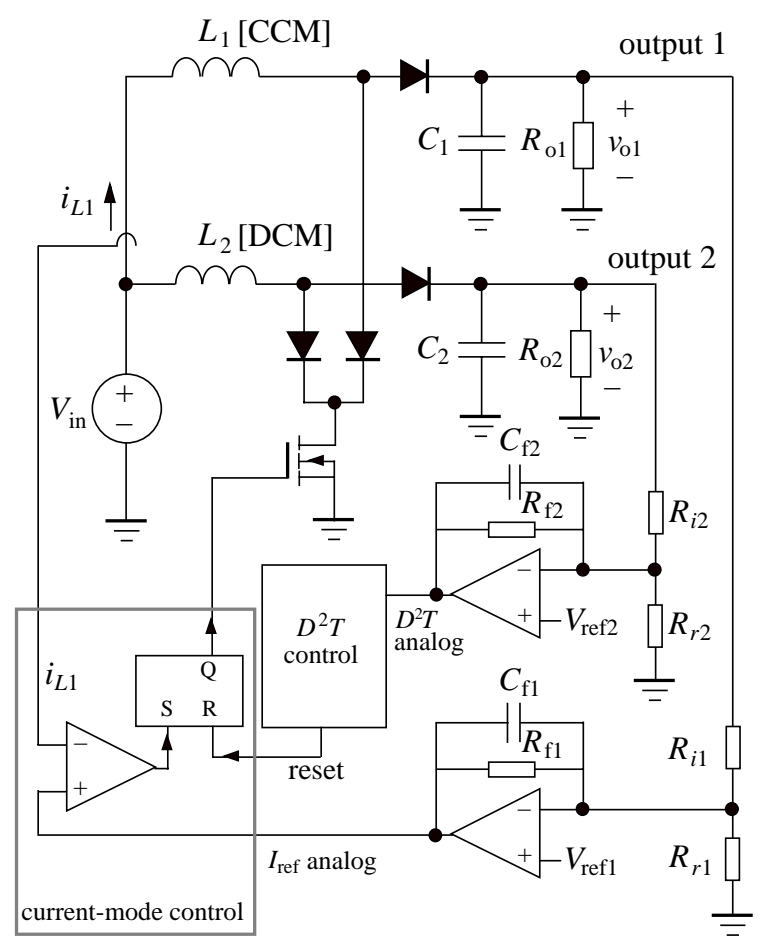

Fig. 3. Schematic of dual-output boost regulator under combined currentmode $D^{2} T$ control. Details of the $D^{2} T$ control block is shown in Fig. 4 .

condition forces

$$
p(D T)=r(T) \Rightarrow a(D T)^{2}=b T \Rightarrow D^{2} T=b / a .
$$

Hence, the quantity $D^{2} T$ can be made controllable by varying $b / a$. A conceptual implementation of this control scheme is shown in Fig. 1 (b). In this scheme, the off-time duration is externally determined, and the control circuit in turn produces a pulse to set the period. Thus, the proposed control can be viewed as a frequency modulator which, for each cycle, maintains a constant $D^{2} T$. If the current sources (i.e., $a$ and b) are controllable, the quantity $D^{2} T$ can be modulated via feedback.

\section{Local Stability of Combined CurRent-Mode AND $D^{2} T$ CONTROL}

Our interest in this section is to study the local stability of the proposed control scheme when the off-time duration is pre-determined by a current-mode control scheme. Essentially, in a typical continuous-mode $\mathrm{dc} / \mathrm{dc}$ converter under currentmode control, the turn-off instant is determined by comparing the inductor current with a reference level. Thus, effectively, this conventional scheme is controlling the on-time duration. In our scheme, however, we control the off-time duration instead. As we will see, this has an important implication on the stability. In brief, the switch is turned on at the instant the inductor current descends to a reference level. The turnoff instant, moreover, is determined by our proposed $D^{2} T$ control. In other words, the repetition period is controlled by the $D^{2} T$ controller. The stability issue therefore involves the consideration of the stability of the current-mode control with off-time duration being pre-determined and the period being controlled by the $D^{2} T$ scheme.

First, referring to Fig. 2, we can write the inductor current values at the start and end of a period as

$$
\begin{aligned}
& i_{1}=I_{\text {ref }}+\left(m_{c}+m_{2}\right) T_{2} \\
& i_{2}=I_{\text {ref }}+m_{c} T_{2}+m_{1} T_{1}
\end{aligned}
$$

where $T_{1}$ is the on-time duration, $T_{2}$ is the off-time duration, $m_{1}$ is the on-time inductor current slope, $m_{2}$ is the offtime inductor current slope, and $m_{c}$ is the compensation slope. Note that we consider here the general case where a compensation slope is included in the current-mode control. Upon differentiating (4), we get

$$
\frac{\delta i_{2}}{\delta i_{1}}=\frac{m_{1} \delta T_{1}+m_{c} \delta T_{2}}{\left(m_{c}+m_{2}\right) \delta T_{2}}=\frac{m_{c}+m_{1} \frac{\delta T_{1}}{\delta T_{2}}}{m_{c}+m_{2}} .
$$

Let us now introduce the $D^{2} T$ control to the abovementioned current-mode controlled converter. In the steady state, the aim is to fix the value of $D^{2} T$, i.e.,

$$
\frac{T_{1}^{2}}{T_{1}+T_{2}}=\text { constant }
$$

where the constant in the RHS is equal to the steady-state value of $D^{2} T$. Thus, differentiating (6), we get

$$
\frac{\delta T_{1}}{\delta T_{2}}=\frac{D}{2-D}
$$

Hence, from (5), and using $m_{1} D=m_{2}(1-D)$, we have

$$
\frac{\delta i_{2}}{\delta i_{1}}=\frac{m_{c}+m_{1} \frac{D}{2-D}}{m_{c}+m_{2}}=\frac{m_{c}+m_{2} \frac{1-D}{2-D}}{m_{c}+m_{2}}
$$

Local stability requires that the magnitude of the above expression be less than 1 . Thus, we can see that stability is guaranteed for all $D$ since

$$
0<\frac{1-D}{2-D}<\frac{1}{2} \text { for all } D \in(0,1)
$$

which implies $\left|\delta i_{2} / \delta i_{1}\right|<1$ for all $0<D<1$.

It is interesting to note that the stability of the system is unaffected even when the compensation ramp is zero. In other words, the $D^{2} T$ control inherently stabilizes the current-mode control, eliminating the need for ramp compensation. 

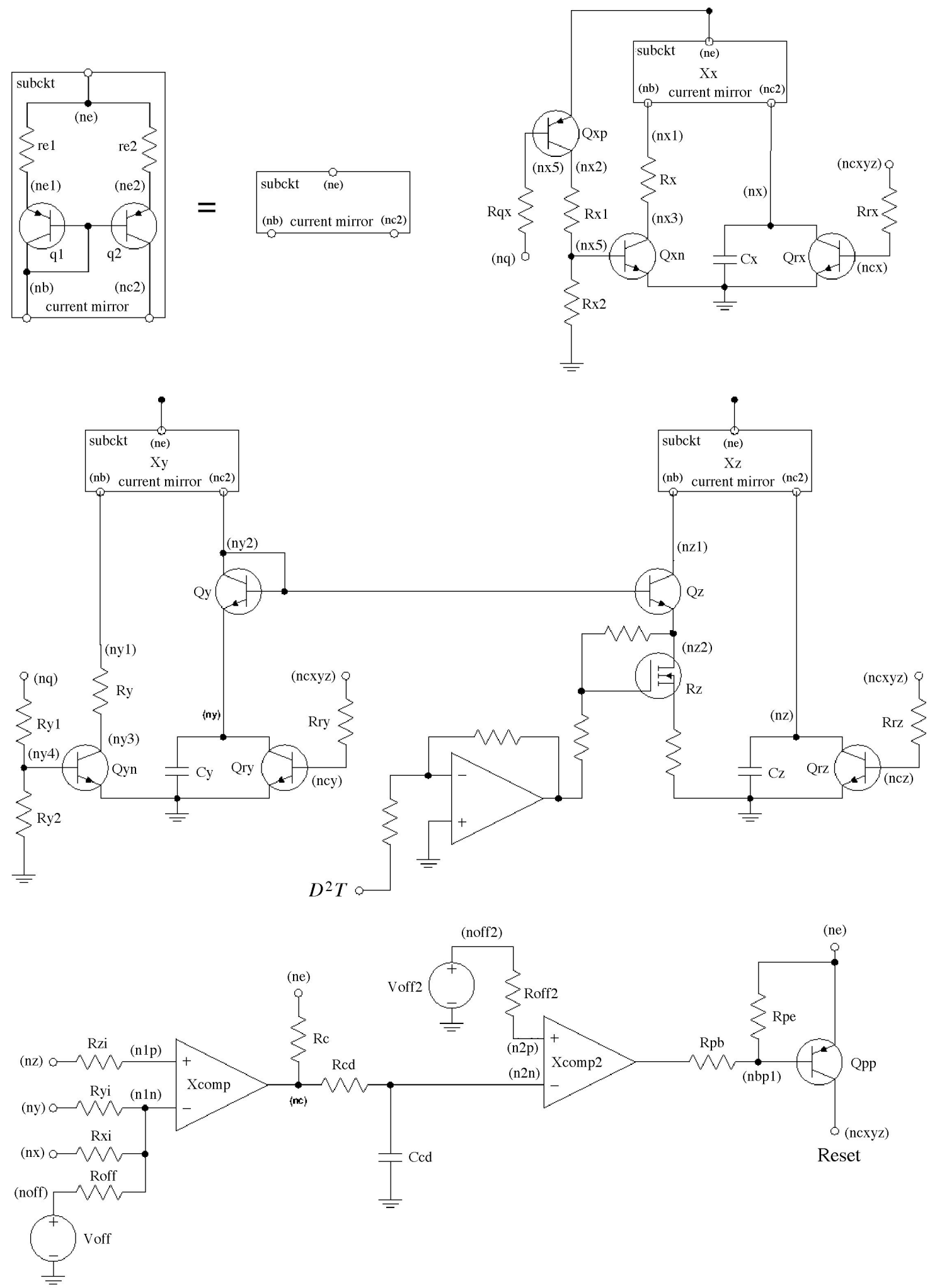

Fig. 4. Detailed circuit schematic of the $D^{2} T$ control.

\section{EXPERIMENTAL VERIFICATION}

In this section we present a single-switch two-output boost regulator, as outlined previously in the Introduction. Figs. 3 and 4 show the schematic of the system and the detailed circuit of the $D^{2} T$ controller, respectively. In brief, this regulator consists of a CCM boost converter and a DCM boost converter, sharing one common switch. The circuit design aspect has been studied extensively by Sebastián et al. [1], [3]. Here, we focus on the application of the proposed combined currentmode and $D^{2} T$ control for simultaneous regulation of the two outputs, and verify the control function with an experimental 


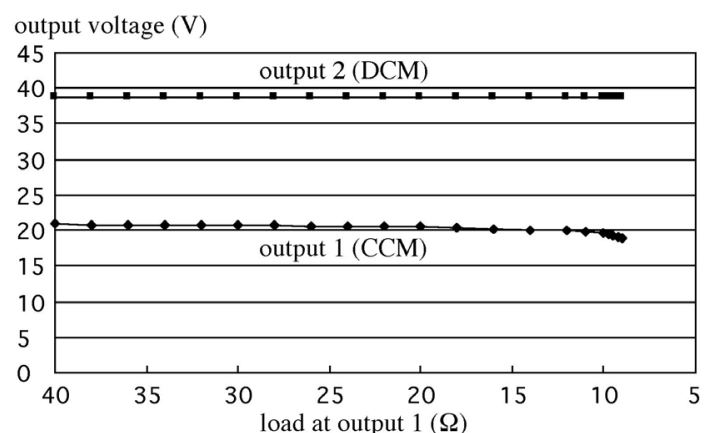

(a)

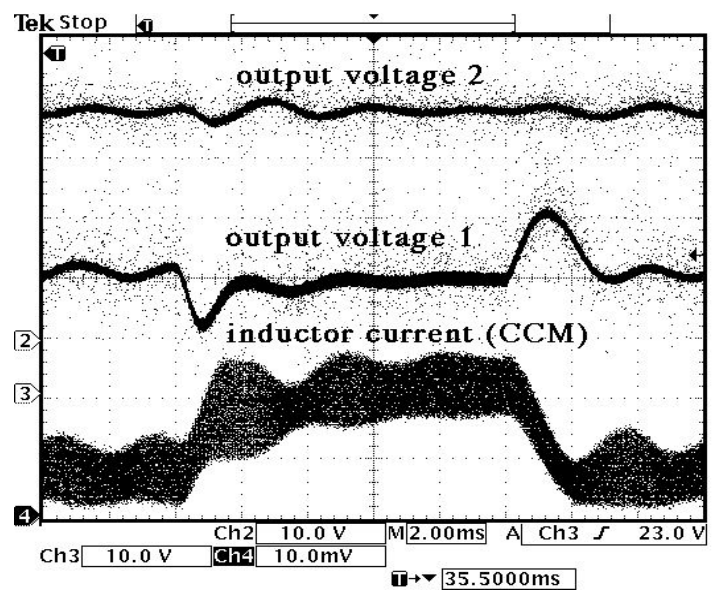

(c)

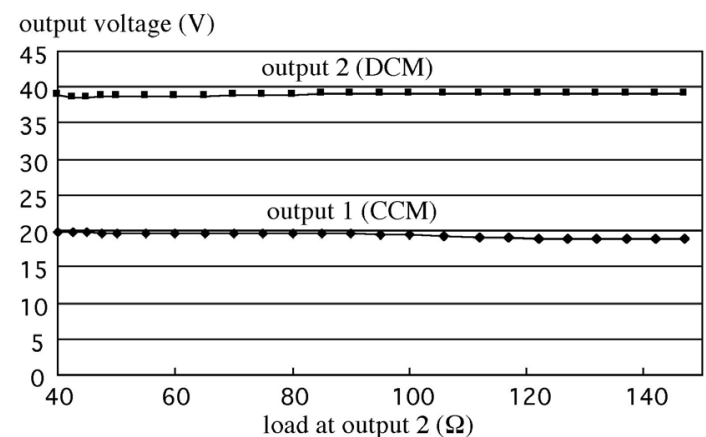

(b)

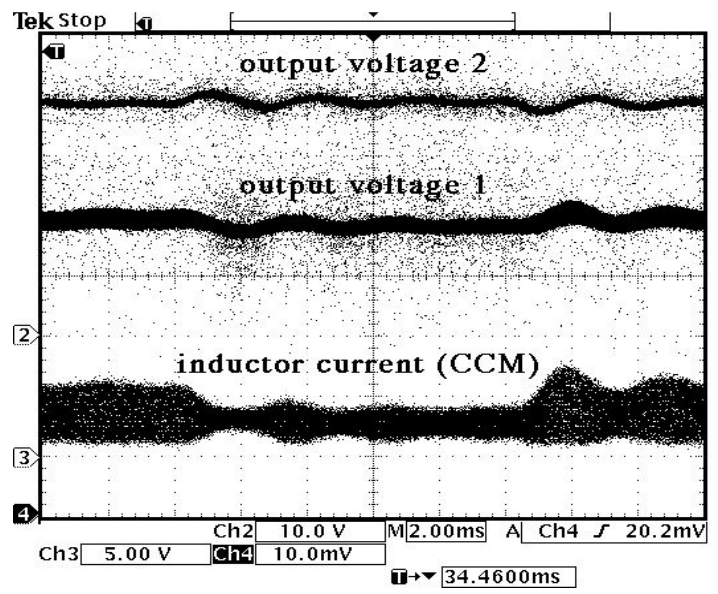

(d)

Fig. 5. Experimentally measured steady-state output voltages versus load variation in output 1, with load at output 2 fixed at $60 \Omega$; (b) steady-state output voltages versus load variation in output 2, with load at output 1 fixed at $10 \Omega$; (c) transient responses for step changing loads with $R$ o1 changing from $10 \Omega$ to $35 \Omega$ at $t=4 \mathrm{~ms}$, and back to $10 \Omega$ at $t=14 \mathrm{~ms}$; (d) transient responses for step changing loads with $R o_{o 2}$ changing from $50 \Omega$ to $100 \Omega$ at $t=4.2 \mathrm{~ms}$, and back to $50 \Omega$ at $t=14.2 \mathrm{~ms}$. Vertical scale: $10 \mathrm{~V} / \mathrm{div}$ and $2 \mathrm{~A} / \mathrm{div}$. Horizontal scale: $2 \mathrm{~ms} / \mathrm{div}$.

TABLE I

COMPONENT AND PARAMETER VALUES OF EXPERIMENTAL PROTOTYPE

\begin{tabular}{lr}
\hline Component/parameter & Value \\
\hline \hline Inductance 1 (CCM), $L_{1}$ & $100 \mu \mathrm{H}$ \\
Inductance 2 (DCM), $L_{2}$ & $20 \mu \mathrm{H}$ \\
Output capacitance 1, $C_{1}$ & $47 \mu \mathrm{F}$ \\
Output capacitance 2, $C_{2}$ & $100 \mu \mathrm{F}$ \\
Input voltage, $V_{\text {in }}$ & 12 to $16 \mathrm{~V}$ \\
Load resistance 1, $R_{o 1}$ & 10 to $40 \Omega$ \\
Load resistance 2, $R_{o 2}$ & 40 to $147 \Omega$ \\
Power MOSFET & IRFP 360 \\
Frequency & 20 to $100 \mathrm{kHz}$ \\
\hline
\end{tabular}

prototype. In the experiment, discrete components are used to construct the the $D^{2} T$ control block, as shown in Fig. 1 (b). Component and parameter values of the prototype are summarized in Table I.

It is worth noting that the inductor current of the CCM converter is referenced at the turn-on instant, and hence its peak is generally unlimited (actually determined by the $D^{2} T$ control). It is thus necessary to impose a peak limiter to limit the maximum switching period. This arrangement has been incorporated in our study.

Several tests have been performed to verify the operation of the proposed control. First of all, the steady-state output characteristics characteristics have been obtained by plotting the output voltages against load variations. Measured results are

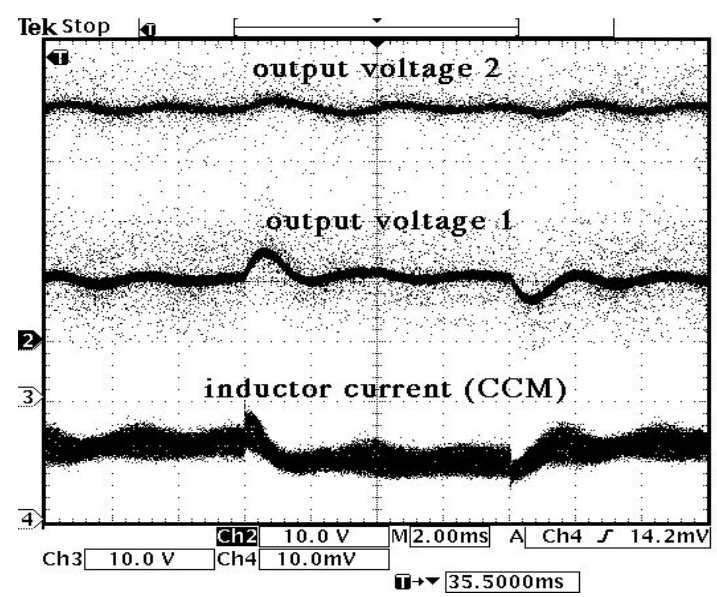

Fig. 6. Output transient responses for step changing input voltage. At $t=$ $6 \mathrm{~ms}, V_{\text {in }}$ steps up from $12 \mathrm{~V}$ to $16 \mathrm{~V}$, and at $t=14 \mathrm{~ms}$, it steps down back to $12 \mathrm{~V}$. Vertical scale: $10 \mathrm{~V} / \mathrm{div}$ and $2 \mathrm{~A} / \mathrm{div}$. Horizontal scale: $2 \mathrm{~ms} / \mathrm{div}$.

shown in Figs. 5 (a) and (b). Then, the transient performance has been evaluated, including input regulation, load regulation and cross regulation. Results are shown in Figs. 5 (c)-(d) and 6. In Figs. 5 (c)-(d), the transient responses are recorded when the load resistances are stepped. Note that Figs. 5 (c)-(d) reflect very satisfactory self load regulation performance as well as cross regulation performance, i.e., transient of $v_{o 1}$ (or 
$v_{o 2}$ ) when $R_{o 2}$ (or $R_{o 1}$ ) is stepped. In Fig. 6, the transient responses are recorded when the input voltage is stepped.

\section{CONCLUSION}

In a dual-output voltage regulator, where two dc/dc converters (one operating in CCM and the other in DCM) are sharing one switch, generic current-mode control can be achieved by applying conventional current-mode control to the CCM converter and a $D^{2} T$ programming control to the DCM converter. In this paper we propose a strategy for controlling the $D^{2} T$ quantity, whereby both outputs can be tightly regulated. Satisfactory cross regulation is possible by virtue of the CCM converter being insensitive to frequency changes and the DCM converter being directly current-programmed. Clearly, the concept of direct $D^{2} T$ control can also be applied to other applications where one switch is required to achieve two functions, e.g., in single-switch single-stage PFC power supplies.

\section{ACKNOWLEDGMENT}

This work is supported by Hong Kong Polytechnic University under Grant G-T662.

\section{REFERENCES}

[1] J. Sebastián and J. Uceda, "Double converter: a fully regulated two output dc-to-dc converter," IEEE PESC Rec., pp. 117-126, 1985

[2] J. Sebastián, J. Uceda, M. Rico, M.A. Pérez and F. Aldana, "A complete study of the double forward-flyback converter," IEEE PESC Rec., pp. 142-149, 1988.

[3] T. Charanasomboon, M.J. Devaney and R.G. Hoft, "Single switch dual output dc/dc converter performance," IEEE Trans. Power Electron., vol. 5, no. 2, pp. 241-245, 1990.

[4] P.T. Tang and C.K. Tse, "Design and implementation of a fully-regulated four-output two-switch one-controller power module," IEEE PESC Rec., pp. 823-828, June 1996.

[5] R. Redl and N.O. Sokal, "Current-mode control, five different types, used with the three basic classes of power converters," IEEE PESC Rec., pp. 771-775, 1985.

[6] P.R. Severns and E.J. Bloom, Modern Switching DC-to-DC Power Converter Circuits, New York: Van Nostrand, 1985. 